\title{
New and corrected records on distribution of Finnish Heteroptera
}

\section{Tapio Lammes \& Veikko Rinne}

Tapio Lammes, Sorolaisenkatu 6, FIN-21200 Raisio, Finland

Veikko Rinne, Zoological Museum, University of Turku, FIN-20500 Turku Finland

We have recently published maps of the provincial distribution of Finnish Heteroptera (Lammes $\&$ Rinne 1990). Careful reading of the maps has revealed some missplaced dots, which are corrected in this article. Numerous new provincial records are also listed.

Most of the specimens are deposited in our personal collections or in Zoological Museum of Turku University.

\section{New records}

Collectors: $\mathrm{TL}=\mathrm{T}$. Lammes, VR $=\mathrm{V}$. Rinne.

$V$ :

Aelia klugi Hahn: Kiikala, old airport, 6710:315, 25.7.1991, TL

Agramma tropidopterum Flor: Marttila, Karhunperänrahka, 6723:283, 13.8.1991, VR

Trigonotylus fuscitarsis Lammes: Kiikala, 6709: 316, 25.7.1991, VR

St:

Anthocoris limbatus Fieber: Huittinen, Kyttälänhaara, 6800:261, 27.7.1991, TL \& VR

Holcostethus vernalis (Wolff): Rauma commune, Sorkka, 6796:210, 27.8.1991, TL

Lygocoris rhamnicola (Reuter): Huittinen, Kyttälänhaara, 6800:261, 27.7.1991, TL \& VR

Trapezonotus desertus Seidenstücker: Alastaro, Virttaankangas, 6771:266 and Oripää, Säkylänharju, 6771:262, 9.6.1991, TL
EH:

Plagiognathus vitellinus (Scholtz): Jokioinen, manor of Jokioinen, 6749:308, 28.7.1991, TL

$E P$ :

Corixa dentipes (Thomson): Ilmajoki, Alajoki, 6972:277, 1.8.1990, V.-M. Mukkala (nymph)

Macrotylus cruciatus (R.F.Sahlberg): Kauhajoki, Koskenkylä, 6930:251, 8.7.1991, TL (5th instar nymphs on Geranium sylvaticum)

PH:

Orthops campestris (Linnaeus): Ähtäri, Ohraniemi, 6953:348, 4.7.1991, TL

PS:

Aradus crenaticollis R.F.Sahlberg: Nilsiä, 70183: 5523, 16.6.1988, P. Rassi

PK:

Chlamydatus opacus (Zetterstedt): Valtimo, village, 7064:591, 2.8.1991, TL

C. wilkinsoni (Douglas \& Scott): Nurmes, Jokikylä, 7054:596, 3.8.1991, TL

Dicyphus constrictus (Boheman): Kitee, Tervavaara, 6892:645, 5.8.1991, TL

Megaloceraea recticornis (Geoffroy): eg. Kitee, Papinniemi, 6883:655, 4.8.1991, TL

Megalocoleus pilosus (Schrank): eg. Valtimo, village, 7064:591, 2.8.1991, TL

Micracanthia marginalis (Fallén): Valtimo, Halmejärvi, 7077:588, 2.8.1991, TL

Nabis ericetorum Scholtz: Kitee, Papinniemi crossroads, 6887:653, 4.8.1991, TL 
N. rugosus (Linnaeus): Kitee, Juurikka, 6880:658, 4.8.1991, TL

Orthotylus nassatus (Fabricius): Kesälahti, railway station, 6867:647, 5.8.1991, TL

O. virens (Fallén): Kitee, Tervavaara, 6892:645, 5.8.1991, TL

Philomyrmex insignis R.F.Sahlberg: Liperi, 695:62, 1.5.1990, P.Turunen

Phimodera lapponica (Zetterstedt): Kitee, Papinniemi crossroads, 6887:653, 4.8.1991, TL

Phytocoris intricatus Flor: Valtimo, village, 7064: 591, 2.8.1991, TL

P. pini Kirschbaum: Kesälahti, railway station, 6867:647, 5.8.1991, TL

P. populi (Linnaeus): Kesälahti, railway station, 6867:647, 5.8.1991, TL

Pilophorus cinnamopterus (Kirschbaum): eg. Valtimo, Halmejärvi, 7077:588, 2.8.1991, TL

Plagiognathus albipennis (Fallén): Kitee, Juurikka, 6880:658, 4.8.1991, TL

Psallus ambiguus (Fallén): Kitee, Papinniemi crossroads, 6887:653, 4.8.1991, TL

$P$. graminicola (Zetterstedt): Valtimo, Halmejärvi, 7077:588, 2.8.1991, TL

Stenotus binotatus (Fabricius): Kitee, Puhos, 6889:653, 3.8.1991, TL

Stictopleurus punctatonervosus (Goeze): Kitee, Juurikka, 6880:658, 4.8.1991, TL

Temnostethus gracilis (Horváth): Kitee, Juurikka, 6880:658, 4.8.1991, TL

$K P$ :

Calocoris biclavatus (Herrich-Schäffer): Vimpeli, Koskela, 7076:347, 5.7.1991, TL (5th instar nymphs)

Ischnocoris angustulus (Boheman): Vimpeli, Hallapuro, 7010:352, 5.7.1991, TL
Macrotylus cruciatus (R.F.Sahlberg): Alajärvi, Koskenvarsi, 6990:335, 7.7.1991, TL

Nabis inscriptus (Kirby): Vimpeli, Hallapuro, 7010:352, 5.7.1991, TL

Placochilus seladonicus (Fallén): Alajärvi, Koivula, 6984:334, 7.7.1991, TL (5th instar nymphs on Knautia arvensis)

PeP:

Dicyphus globulifer (Fallén): Keminmaa, Kalkkimaa, 7314:384, 1.7.1991, VR

SoL:

Aradus pictus Bärensprung: Sodankylä, 75825: 5159, 4.7.1989, P. Rassi

\section{Corrections:}

Aradus laeviusculus Reuter: add $P e P$

A. pictus Bärensprung: delete $P e P$, add $O P$ Atractotomus morio J.Sahlberg: add EP

Capsus wagneri Remane: add KiL and SoL

Eremocoris plebeius (Fallén): add EH

Salicarus roseri (Herrich-Schäffer): add ES

Sigara falleni (Fieber): add ES

Strongylocoris niger (Herrich-Schäffer): delete $O P$

Temnostethus pusillus (Herrich-Schäffer): delete $U$, add $A$

Xylocoris formicetorum (Boheman): add $A$

\section{References}

Lammes, T. \& Rinne, V. 1990: Maps of the provincial distribution of Finnish Heteroptera. - Entomol. Fennica 1:209-220. 